\title{
Approximating Matsubara dynamics using the planetary model: tests on liquid water
}

and ice

Michael J. Willatt, ${ }^{1}$ Michele Ceriotti, ${ }^{2}$ and Stuart C. Althorpe ${ }^{\mathrm{a})}{ }^{1}$

1) Department of Chemistry, University of Cambridge, Lensfield Road, Cambridge, CB2 $1 E W, U K$.

2) Laboratory of Computational Science and Modeling, Institute of Materials, École Polytechnique Fédérale de Lausanne, 1015 Lausanne,

Switzerland.

(Dated: 6 December 2017)

Matsubara dynamics is the quantum-Boltzmann-conserving classical dynamics which remains when real-time coherences are taken out of the exact quantum Liouvillian [J. Chem. Phys. 142, 134103 (2015)]; because of a phase-term, it cannot be used as a practical method without further approximation. Recently, Smith et al. [J. Chem. Phys. 142, 244112 (2015)] developed a 'planetary' model dynamics which conserves the Feynman-Kleinert (FK) approximation to the quantum Boltzmann distribution. Here, we show that, for moderately anharmonic potentials, the planetary dynamics gives a good approximation to Matsubara trajectories on the FK potential surface, by decoupling the centroid trajectory from the locally harmonic Matsubara fluctuations, which reduce to a single phase-less fluctuation particle (the 'planet'). We also show that the FK effective frequency can be approximated by a direct integral over these fluctuations, obviating the need to solve iterative equations. This modification, together with use of thermostatted ring-polymer molecular dynamics (TRPMD), allows us to test the planetary model on water (gas-phase, liquid and ice), using the q-TIP4P/F potential surface. The 'planetary' fluctuations give a poor approximation to the rotational/librational bands in the infrared spectrum, but a good approximation to the bend and stretch bands, where the fluctuation lineshape is found to be motionally narrowed by the vibrations of the centroid.

a) Corresponding author: sca10@cam.ac.uk 


\section{INTRODUCTION}

Liquid water is an example of a condensed-phase system in which the nuclear statistics are quantum Boltzmann, but the nuclear dynamics are (expected to be) mainly classical, owing to rapid decoherence times $\frac{112}{12}$ 'Matsubara dynamics' is a recently derived theory which, to the best of our knowledge, gives the first consistent explanation of how such a classical dynamics emerges from the exact quantum dynamics, when motion is confined to one electronic potential energy surface. It shows that quantum-dynamical effects arise solely from non-smooth components of the imaginary-time Feynman paths in the quantum Boltzmann distribution. When these 'jagged' components can be mean-fielded out, the smooth paths that remain are found to obey a delocalised classical dynamics which preserves the quantum Boltzmann distribution.

The difficulty with Matsubara dynamics is finding a way to implement the theory practically, on account of phases which appear in the quantum statistics. At initial time $t=0$, the phases can be integrated out to give the standard 'ring-polymer' representation, $\stackrel{4}{\text { but at }}$ $t>0$, the phases remain, giving a sign-problem. It seems likely that an exact implementation of Matsubara dynamics will never be found, and that the best use to make of it is as a starting point from which to develop and critique approximate methods of combining quantum Boltzmann statistics with classical dynamics.

A range of such methods has existed for some time, and it is possible to rationalise each of them in terms of Matsubara dynamics. The oldest method is the 'classical Wigner' or LSC-IVR (linear semiclassical initial-value representation) approach, ${ }^{5-17}$ which is equivalent to adding the classical dynamics of the jagged modes to the Matsubara dynamics; this additional approximation allows most of the phase to be integrated out, at the expense of no longer conserving the quantum Boltzmann distribution. A promising recent method is the path-integral Liouvillian approach,,$\sqrt[8]{ }$ which corrects for the non-quantum-Boltzmann conservation of LSC-IVR, and has recently been applied to gas-phase molecules.$^{9}$ For condensedphase systems, the methods most commonly used are (thermostatted) ring-polymer molecular dynamics $[(\mathrm{T}) \mathrm{RPMD}]^{10} \frac{14}{14}$ and centroid molecular dynamics (CMD) $\stackrel{15}{17}$ Both methods were originally obtained heuristically, but can also be obtained by making (uncontrolled, but quantum-Boltzmann-conserving) approximations to Matsubara dynamics. $\underline{[}$

In this article, we examine the relation to Matsubara dynamics of another heuristically- 
obtained method - the Feynman-Kleinert (FK) quasi-classical Wigner method, or 'planetary model' 18119 This model is exact in the harmonic limit, not just for the dynamics of the centroid (as is true also for (T)RPMD and CMD), but also for the dynamics of fluctuations about the centroid. The planetary model describes these fluctuations by means of a single particle ('the planet') which follows locally harmonic motion, with a centroid-dependent frequency (see Fig. 1). Unlike RPMD and CMD, the planetary model therefore has the potential to treat non-linear time-correlation functions (TCFs), which (at low enough temperatures) depend explicitly on the dynamical fluctuations. ${ }^{20}$ [Note that a related method ${ }^{21}$ was developed at around the same time as the planetary model, but this uses a different type of locally harmonic force on the fluctuation particle (called a 'pseudoparticle'), 22 and has so far been restricted to static applications.]

In this article, we focus on linear TCFs, since these allow us to benchmark the planetary model against TRPMD and CMD results. This is of course not as good as comparing with exact quantum results, but is the best that we can do for condensed-phase systems. We use the planetary model to approximate the standard TCF

$$
C_{A B}(t)=\frac{1}{Z} \operatorname{Tr}\left[e^{-\beta \hat{H}} \hat{A} e^{i \hat{H} t / \hbar} \hat{B} e^{-i \hat{H} t / \hbar}\right],
$$

and TRPMD and CMD to approximate the Kubo TCF ${ }^{23}$

$$
\widetilde{C}_{A B}(t)=\frac{1}{\beta Z} \int_{0}^{\beta} d \lambda \operatorname{Tr}\left[e^{-\lambda \hat{H}} \hat{A} e^{-(\beta-\lambda) \hat{H}} e^{i \hat{H} t / \hbar} \hat{B} e^{-i \hat{H} t / \hbar}\right],
$$

(where $\beta=1 / k_{\mathrm{B}} T, Z$ is the quantum partition function and $\hat{H}$ the system Hamiltonian). We compare the two TCFs via their frequency spectra $G_{A B}(\omega)$ and $\widetilde{G}_{A B}(\omega)$, using the well-known expression ${ }^{10}$

$$
\widetilde{G}_{A B}(\omega)=\frac{1-e^{-\beta \hbar \omega}}{\beta \hbar \omega} G_{A B}(\omega),
$$

Since $C_{A B}(t)$ is often dominated by the fluctuation dynamics (e.g. in the stretch-band of liquid water) and $\widetilde{C}_{A B}(t)$ depends explicitly only on the centroid dynamics, it follows that this comparison is equivalent to checking that the planetary model of the fluctuation dynamics is consistent with the TRPMD or CMD approximation to the centroid dynamics.

The article is structured as follows: After reviewing Matsubara dynamics in Sec. II, we show in Sec. III that the planetary model is an approximation to Matsubara dynamics within the FK approximation, whereby one neglects the influence of the dynamical fluctuations on 
the centroid trajectory. We find that this approximation is sufficient when the dynamics is moderately anharmonic, which is when the FK approximation itself can be expected to hold. In Sec. IV we introduce some practical steps that allow the FK effective frequency to be evaluated directly for multi-dimensional systems. These allow us, in Sec. V, to make comparisons with TRPMD and CMD (as just described) for the infrared spectra of water (gas-phase, liquid and ice), calculated using the q-TIP4P/F potential and assuming a linear dipole-moment operator. Section VI concludes the article.

\section{SUMMARY OF MATSUBARA DYNAMICS}

\section{A. Matsubara modes}

To explain Matsubara dynamics we must first summarise the role of Matsubara modes in quantum Boltzmann statistics. To simplify the algebra, we focus throughout on one-

dimensional systems with position operator $\hat{q}$ and potential energy $V(\hat{q})$, and we assume that the operators $\hat{A}$ and $\hat{B}$ are functions of $\hat{q}$. These results are easy to generalize (and we give the multi-dimensional generalization of the planetary model in Sec. IV).

At $t=0$, the Kubo time-correlation function can be evaluated exactly by path-integral theory, as a classical phase-space average over an extended 'ring-polymer' space! $\underline{1024} 27$

$$
\widetilde{C}_{A B}(0)=\lim _{N \rightarrow \infty} \frac{1}{(2 \pi \hbar)^{N} Z} \int d \mathbf{p} \int d \mathbf{q} e^{-\beta R_{N}(\mathbf{p}, \mathbf{q})} A_{N}(\mathbf{q}) B_{N}(\mathbf{q}),
$$

where $\beta_{N}=\beta / N, \int d \mathbf{p} \equiv \int_{-\infty}^{\infty} d p_{1} \ldots \int_{-\infty}^{\infty} d p_{N}$ and similarly for $\int d \mathbf{q}$, and

$$
\begin{aligned}
R_{N}(\mathbf{p}, \mathbf{q}) & =T_{N}(\mathbf{p}, \mathbf{q})+U_{N}(\mathbf{q}) \\
T_{N}(\mathbf{p}, \mathbf{q}) & =\frac{1}{N} \sum_{i=1}^{N} \frac{p_{i}^{2}}{2 m}+\frac{m}{2\left(\beta_{N} \hbar\right)^{2}}\left(q_{i+1}-q_{i}\right)^{2} \\
U_{N}(\mathbf{q}) & =\frac{1}{N} \sum_{i=1}^{N} V\left(q_{i}\right)
\end{aligned}
$$

with $A_{N}(\mathbf{q}), B_{N}(\mathbf{q})$ defined analogously to $U_{N}(\mathbf{q})$.

We can apply a discrete Fourier transform to the bead coordinates $\mathbf{q}$ (and $\mathbf{p}$ ), which is equivalent to transforming to the normal modes of a free ring-polymer (i.e. diagonalising the 
Hessian in $\left.T_{N}(\mathbf{p}, \mathbf{q})\right)$. These normal modes are

$$
Q_{n}=\frac{1}{N} \sum_{l=1}^{N} T_{l n} q_{l}, \quad n=0, \pm 1, \ldots, \pm(N-1) / 2
$$

with

$$
T_{l n}= \begin{cases}1 & n=0 \\ \sqrt{2} \sin (2 \pi l n / N) & n=1, \ldots,(N-1) / 2 \\ \sqrt{2} \cos (2 \pi l n / N) & n=-1, \ldots,-(N-1) / 2\end{cases}
$$

and the frequencies $\operatorname{arc}^{28}$

$$
\omega_{n}^{\prime}=\frac{2}{\beta_{N} \hbar} \sin \left(\frac{n \pi}{N}\right)
$$

Taking the limit $N \rightarrow \infty$, we obtain the 'Matsubara modes', which are all modes $|n| \leq$ $(M-1) / 2$ for which $M \ll N$, such that the frequencies $\omega_{n}$ are given by

$$
\omega_{n}=\lim _{N \rightarrow \infty} \omega_{n}^{\prime}=\frac{2 n \pi}{\beta \hbar}, \quad|n| \leq(M-1) / 2 .
$$

The significance of the Matsubara modes is that a superposition of them gives a distribution of positions $q(\tau)$ (and similarly momenta $p(\tau)$ ) which is a smooth and continuous function of imaginary time,

$$
q(\tau)=Q_{0}+\sqrt{2} \sum_{n=1}^{(M-1) / 2} Q_{n} \sin \left(\omega_{n} \tau\right)+Q_{-n} \cos \left(\omega_{n} \tau\right), \quad 0 \leq \tau<\beta \hbar
$$

satisfying $q(\tau+\beta \hbar)=q(\tau)$. Inclusion of the remaining $N-M$ 'non-Matsubara' modes

produces discontinuous or 'jagged' distributions which in general resemble random walks. ${ }^{26 \mid 29}$

Taking the limit $M \rightarrow \infty, M \ll N$, allows one to integrate out the non-Matsubara modes analytically, giving

$$
\widetilde{C}_{A B}(0)=\lim _{M \rightarrow \infty} \frac{1}{(2 \pi \hbar)^{M} Z_{M}} \int d \mathbf{P} \int d \mathbf{Q} e^{-\beta R_{M}(\mathbf{P}, \mathbf{Q})} A_{M}(\mathbf{Q}) B_{M}(\mathbf{Q}),
$$

where

$$
\begin{aligned}
R_{M}(\mathbf{P}, \mathbf{Q}) & =U_{M}(\mathbf{Q})+\sum_{n=-(M-1) / 2}^{(M-1) / 2} \frac{P_{n}^{2}}{2 m}+\frac{m}{2} \omega_{n}^{2} Q_{n}^{2} \\
U_{M}(\mathbf{Q}) & =\frac{1}{\beta \hbar} \int_{0}^{\beta \hbar} d \tau V[q(\tau)] \\
Z_{M} & =\frac{1}{(2 \pi \hbar)^{M}} \int d \mathbf{P} \int d \mathbf{Q} e^{-\beta R_{M}(\mathbf{P}, \mathbf{Q})}
\end{aligned}
$$


with $A_{M}(\mathbf{Q})$ and $B_{M}(\mathbf{Q})$ defined analogously to $U_{M}(\mathbf{Q})$. Note that this expression is formally exact, because the (very large) contribution of the non-Matsubara modes to the integral of Eq. (4) cancels out with their contribution to $Z$, leaving $Z_{M}$ in the denominator of Eq. (11).

The most important Matsubara mode is the centroid $Q_{0}$ which is the centre of mass of the distribution $q(\tau)$. The centroid is the only mode that survives in the high temperature limit $\beta \rightarrow 0$. The free energy of the centroid (neglecting the $Q_{0}$-independent non-Matsubara contribution) is

$$
F_{M}\left(Q_{0}\right)=-\frac{1}{\beta} \ln z_{M}\left(Q_{0}\right)
$$

where

$$
z_{M}\left(Q_{0}\right)=\frac{1}{(2 \pi \hbar)^{M-1}} \int^{\prime} d \mathbf{P} \int^{\prime} d \mathbf{Q} e^{-\beta R_{M}(\mathbf{P}, \mathbf{Q})} .
$$

and the primes denote integration over the modes $P_{n}, Q_{n},|n|>0$. The modes $Q_{n},|n|>0$ describe quantum-Boltzmann fluctuations around $Q_{0}$ with radius of gyration

$$
a^{2}\left(Q_{0}\right)=\lim _{M \rightarrow \infty} \frac{1}{(2 \pi \hbar)^{M-1} z_{M}\left(Q_{0}\right)} \int^{\prime} d \mathbf{P} \int^{\prime} d \mathbf{Q} e^{-\beta R_{M}(\mathbf{P}, \mathbf{Q})} \sum_{n}^{\prime} Q_{n}^{2} .
$$

and the modes $P_{n},|n|>0$ fluctuate similarly around $P_{0}$.

\section{B. Matsubara dynamics}

Equation (11) is exact (in the limit $M \rightarrow \infty, M \ll N)$ and is the result of integrating out the jagged modes in $\widetilde{C}_{A B}(t)$ at $t=0$. Matsubara dynamics is the approximate dynamics that results if one assumes that the jagged modes can be similarly integrated out for $t>0$, which is equivalent to decoupling the dynamics of the smooth Matsubara modes from the dynamics of the jagged modes. The resulting Matsubara-dynamics approximation to $\widetilde{C}_{A B}(t)$ is 3

$$
\widetilde{c}_{A B}(t)=\lim _{M \rightarrow \infty} \frac{1}{(2 \pi \hbar)^{M} Z_{M}} \int d \mathbf{P} \int d \mathbf{Q} e^{-\beta\left[H_{M}(\mathbf{P}, \mathbf{Q})-i \theta_{M}(\mathbf{P}, \mathbf{Q})\right]} A_{M}(\mathbf{Q}) B_{M}\left(\mathbf{Q}_{t}\right),
$$

with

$$
\begin{aligned}
H_{M}(\mathbf{P}, \mathbf{Q}) & =\frac{\mathbf{P}^{2}}{2 m}+U_{M}(\mathbf{Q}) \\
\theta_{M}(\mathbf{P}, \mathbf{Q}) & =\sum_{n=-(M-1) / 2}^{(M-1) / 2} P_{n} \omega_{n} Q_{-n},
\end{aligned}
$$


where $\mathbf{Q}_{t}$ follows a classical trajectory generated by the Hamiltonian dynamics of $H_{M}(\mathbf{P}, \mathbf{Q})$. The fact that Matsubara dynamics is classical is (in our opinion) a remarkable result, since no classical approximation is introduced explicitly when obtaining Eq. (16); it just happens that the dynamics of the Matsubara modes become classical when they are decoupled from the non-Matsubara modes. $\frac{330}{30}$

The phase $\theta_{M}(\mathbf{P}, \mathbf{Q})$ converts what would otherwise be a classical Boltzmann distribution (in $M$ dimensions) into a quantum Boltzmann distribution (in one dimension). It is easy to show that the Matsubara dynamics conserves $\theta_{M}(\mathbf{P}, \mathbf{Q})$ and thus conserves the quantum Boltzmann distribution. ${ }^{[3}$ At $t=0$, analytical continuation can be used to convert $\theta_{M}(\mathbf{P}, \mathbf{Q})$ into the ring-polymer spring term $m \sum_{n}^{\prime} \omega_{n}^{2} Q_{n}^{2} / 2$, proving that $\widetilde{c}_{A B}(0)=\widetilde{C}_{A B}(0)$. But at $t>0, \theta_{M}(\mathbf{P}, \mathbf{Q})$ cannot be removed by analytic continuation, nor integrated out, unless the dynamics is harmonic. This means that Matsubara dynamics cannot be used as a practical method without making further approximations. Two such approximations are (T)RPMD and CMD. Both these approximations appear to be uncontrolled ${ }^{4}$ but they often give a reasonable description of the dynamics of the centroid. However neither (T)RPMD and CMD is able to treat the dynamics of the fluctuations around the centroid, described by $Q_{n \neq 0, t} \cdot \sqrt{4}$

In Sec. III, we will show that the planetary model gives a good approximation to the dynamics of $Q_{n \neq 0, t}$, provided the forces are reasonably harmonic. This will require us to consider the Matsubara approximation to the standard time-correlation function $C_{A B}(t)$. It is quite involved to derive this quantity for a general $\hat{A}$ and $\hat{B}$, and this task will be left for future work ${ }^{311}$ However, for the special case considered here, that $\hat{A}$ and $\hat{B}$ depend linearly on $\hat{q}$, it follows from Eq. (10) that the Matsubara approximation to the real part of $C_{q q}(t)$, $C_{q q}^{[R]}(t)$, can be obtained by replacing $A_{M}(\mathbf{Q})$ and $B_{M}(\mathbf{Q})$ in Eq. 16 by $\bar{q}$ and $\bar{q}_{t}$, where $\bar{q}=q(\tau=0)$, and $\bar{q}_{t}=q_{t}(\tau=0){ }^{[32}$ The resulting Matsubara approximation to $C_{q q}^{[R]}(t)$ is then

$$
c_{q q}^{[R]}(t)=\lim _{M \rightarrow \infty} \frac{1}{(2 \pi \hbar)^{M} Z_{M}} \int d \mathbf{P} \int d \mathbf{Q} e^{-\beta\left[H_{M}(\mathbf{P}, \mathbf{Q})-i \theta_{M}(\mathbf{P}, \mathbf{Q})\right]} \bar{q} \bar{q}_{t} .
$$

To emphasise the contribution made to $c_{q q}^{[R]}(t)$ by the fluctuation dynamics, we can split $\bar{q}$ 
and $\bar{q}_{t}$ into centroid and fluctuation components,

$$
\begin{aligned}
\bar{q} & =Q_{0}+\tilde{q} \\
\bar{q}_{t} & =Q_{0, t}+\tilde{q}_{t},
\end{aligned}
$$

where, using Eq. (10),

$$
\tilde{q}=\sqrt{2} \sum_{m=1}^{(M-1) / 2} Q_{-m}
$$

The contributions of the fluctuation components $\tilde{q}$ and $\tilde{q}_{t}$ grow with increasing $\beta \hbar \omega$, disappearing entirely in the high-temperature limit, but dominating at sufficiently low temperatures (e.g. 300K, for the OH-stretch band of water-see Sec. V).

\section{THE PLANETARY MODEL AND ITS RELATION TO MATSUBARA DYNAMICS}

\section{A. The Feynman-Kleinert approximation}

The planetary model involves two approximations: the Feynman-Kleinert (FK) approximation, and a model dynamics involving a 'planet' which represents dynamical fluctuations around the centroid. The FK approximation is equivalent to approximating the Matsubara potential $U_{M}(\mathbf{Q})$ by

$$
U_{\mathrm{FK}}(\mathbf{Q})=L\left(Q_{0}\right)+\frac{m}{2} \Omega^{2}\left(Q_{0}\right) \sum_{n}^{\prime} Q_{n}^{2},
$$

(where we have suppressed the $M$-dependence of $\left.U_{\mathrm{FK}}(\mathbf{Q})\right) \stackrel{33}{3}$ The centroid component $L\left(Q_{0}\right)$ and the local frequency $\Omega\left(Q_{0}\right)$ are found by variationally optimising the centroid free-energy $F_{M}\left(Q_{0}\right)$ of Eq. 13 ). In Appendix A (which summarises the results of Feynman and Kleinert's original paper $\left.{ }^{(34}\right)$, it is shown that the variationally optimized $L\left(Q_{0}\right)$ and $\Omega\left(Q_{0}\right)$ satisfy

$$
\begin{aligned}
L\left(Q_{0}\right) & =V_{a^{2}}\left(Q_{0}\right)-\frac{m}{2} \Omega^{2}\left(Q_{0}\right) a^{2} \\
V_{a^{2}}\left(Q_{0}\right) & =\frac{1}{\sqrt{2 \pi a^{2}}} \int_{-\infty}^{\infty} d q V(q) e^{-\left(Q_{0}-q\right)^{2} / 2 a^{2}} \\
\Omega^{2}\left(Q_{0}\right) & =\frac{1}{m} \frac{\partial^{2}}{\partial Q_{0}^{2}} V_{a^{2}}\left(Q_{0}\right),
\end{aligned}
$$


where

$$
a^{2}\left(Q_{0}\right)=\frac{1}{m \Omega^{2} \beta}\left[\frac{\beta \hbar \Omega}{2} \operatorname{coth}(\beta \hbar \Omega / 2)-1\right]
$$

is the radius of gyration of the fluctuations (obtained by substituting $U_{F K}(\mathbf{Q})$ into Eq. (15)).

The 'smeared potential' $V_{a^{2}}\left(Q_{0}\right)$ of Eq. (23) corresponds to integrating the system potential $V\left(Q_{0}+\tilde{q}\right)$ over the locally harmonic Matsubara fluctuations $Q_{n \neq 0}$ (which give a Gaussian distribution in $\tilde{q}$ with variance equal to the square of the radius of gyration $a^{2}$ ). The frequency of these fluctuations $\Omega\left(Q_{0}\right)$ depends on $a^{2}\left(Q_{0}\right)$ through Eq. (25). Hence implementation of the FK approximation requires the iterative solution of the non-linear simultaneous Eqs. (24) and (25) at each value of $Q_{0}$. In Sec. IV, we propose a simple approximation to $V_{a^{2}}\left(Q_{0}\right)$ and $\Omega\left(Q_{0}\right)$, which allows us to bypass this potentially expensive step. But for the time being, we will assume that $V_{a^{2}}\left(Q_{0}\right)$ and $\Omega\left(Q_{0}\right)$ are calculated as given in Eqs. 23) and (24).

\section{B. A first-order approximation to FK fluctuation dynamics}

Since the FK approximation is equivalent to approximating $U_{M}(\mathbf{Q})$ by $U_{\mathrm{FK}}(\mathbf{Q})$, it follows that there is a corresponding FK Matsubara dynamics, with trajectories satisfying

$$
\begin{aligned}
& \dot{Q}_{0}=\frac{P_{0}}{m} \\
& \dot{P}_{0}=-\frac{\partial L\left(Q_{0}\right)}{\partial Q_{0}}-\frac{m}{2} \frac{\partial \Omega^{2}\left(Q_{0}\right)}{\partial Q_{0}} \sum_{n}^{\prime} Q_{n}^{2}
\end{aligned}
$$

and

$$
\begin{aligned}
& \dot{Q}_{n}=\frac{P_{n}}{m} \\
& \dot{P}_{n}=-m \Omega^{2}\left(Q_{0}\right) Q_{n}, \quad n \neq 0,
\end{aligned}
$$

(where we have suppressed the $t$-dependence of $\left(P_{0}, Q_{0}\right)$ and $\left.\left(P_{n}, Q_{n}\right)\right)$. In general, this dynamics is just as difficult to treat as Matsubara dynamics on the exact surface $U_{M}(\mathbf{Q})$, because the time-dependent frequency $\Omega\left(Q_{0, t}\right)$ depends on $Q_{n \neq 0}$ through $Q_{0, t}$. Such nonlinearity makes it impossible to integrate out the phase $\theta_{M}(\mathbf{P}, \mathbf{Q})$.

However, if the potential is not too strongly anharmonic, it seems reasonable to approximate the coupling between $Q_{n \neq 0, t}$ and $Q_{0, t}$ to first-order, allowing $Q_{n \neq 0, t}$ to depend on $Q_{0}$, 
but neglecting the 'back-coupling' whereby $Q_{0, t}$ also depends on $Q_{n \neq 0}$. For example, one could propagate $Q_{0, t}$ using a method such as (T)RPMD or CMD, then insert the resulting time-dependent $\Omega\left(Q_{0, t}\right)$ into Eq. (27), to generate purely harmonic dynamics for $Q_{n \neq 0}$. We tested this first-order approximation on various one-dimensional potentials, using CMD to propagate the centroid trajectories. Typical results are shown in Fig. 2. For moderately anharmonic potentials (in this case a cut through the $\mathrm{OH}$ stretch on the $\mathrm{q}-\mathrm{TIP} 4 \mathrm{P} / \mathrm{F}$ potential) the trajectory followed by the first-order $\tilde{q}_{t}$ is very close to that followed by the exact Matsubara trajectory on the same FK surface. For strongly anharmonic potentials (in this case a cut through a 'Champagne bottle' tangential to the curve), the approximation is very poor. This is only to be expected, as strong anharmonicity is bound to lead to a breakdown of this simple first-order approximation. Given that the exact FK Matsubara dynamics will itself be wrong for such potentials (i.e. one cannot approximate highly anharmonic dynamics by an effective harmonic dynamics), it seems reasonable to suppose that the first-order approximation is sufficient in those regions of the spectrum in which the FK approximation can be trusted. This assumption will be tested numerically in Sec. V.

\section{The planetary model}

The advantage of such a first-order approximation is that it allows the phase $\theta_{M}(\mathbf{P}, \mathbf{Q})$ to be integrated out. One need consider only the dynamics of $\tilde{q}_{t}$, which (being a linear combination of the $Q_{n \neq 0}$ ) obeys

$$
\begin{aligned}
\dot{\tilde{q}} & =\frac{\tilde{p}}{m} \\
\dot{\tilde{p}} & =-m \Omega^{2} \tilde{q}
\end{aligned}
$$

and is a function of only $\left(\tilde{p}, \tilde{q}, P_{0}, Q_{0}\right)$. The phase can then be eliminated by integrating over all linear combinations of $P_{n \neq 0}$ and $Q_{n \neq 0}$ orthogonal to $\tilde{p}$ and $\tilde{q}$ (see Appendix C), to give

$$
c_{q q}^{[\mathrm{R}]}(t) \simeq\left\langle Q_{0} Q_{0, t}\right\rangle_{0}+\left\langle\frac{1}{2 \pi a^{2} m \Omega} \int d \tilde{p} \int d \tilde{q} e^{-\tilde{q}^{2} / 2 a^{2}} e^{-\tilde{p}^{2} / 2 m^{2} \Omega^{2} a^{2}} \tilde{q} \tilde{q}_{t}\right\rangle_{0},
$$

where

$$
\langle\ldots\rangle_{0}=\frac{1}{2 \pi \hbar Z_{\mathrm{FK}}} \int d P_{0} \int d Q_{0} \ldots e^{-\beta P_{0}^{2} / 2 m} e^{-\beta L\left(Q_{0}\right)} \frac{\beta \hbar \Omega / 2}{\sinh (\beta \hbar \Omega / 2)}
$$


and

$$
Z_{\mathrm{FK}}=\frac{1}{2 \pi \hbar} \int d P_{0} \int d Q_{0} e^{-\beta P_{0}^{2} / 2 m} e^{-\beta L\left(Q_{0}\right)} \frac{\beta \hbar \Omega / 2}{\sinh (\beta \hbar \Omega / 2)},
$$

with $a$ given by Eq. 25.

When the dynamics of $Q_{0, t}$ is generated by CMD, Eqs. (28) and (31) almost describe the planetary model of ref. 18, with $\left(\tilde{p}_{t}, \tilde{q}_{t}\right)$ giving the location in phase space of a 'planet' describing dynamical fluctuations about the centroid, as represented in Fig. 1. To obtain exactly the planetary model, we need to modify Eq. 28 to $\frac{18}{18}$

$$
\begin{aligned}
& \dot{\tilde{q}}=\frac{\tilde{p}}{m}+\tilde{q} \frac{d}{d t} \ln (a) \\
& \dot{\tilde{p}}=-m \Omega^{2} \tilde{q}+\tilde{p} \frac{d}{d t} \ln (a \Omega),
\end{aligned}
$$

which ensures that

$$
\frac{d}{d t}\left(\frac{\tilde{q}^{2}}{2 a^{2}}+\frac{\tilde{p}^{2}}{2 m^{2} \Omega^{2} a^{2}}\right) \equiv \frac{1}{a^{2}}\left[\tilde{q} \dot{\tilde{q}}+\frac{\tilde{p} \dot{\tilde{p}}}{m^{2} \Omega^{2}}-\tilde{q}^{2} \frac{d}{d t} \ln a-\frac{\tilde{p}^{2}}{m^{2} \Omega^{2}} \frac{d}{d t} \ln (a \Omega)\right]=0,
$$

i.e. that the trajectories conserve the $(\tilde{p}, \tilde{q})$-distribution of Eq. (29). This final step is ad hoc, but it appears to have a relatively small effect on the trajectory of $\tilde{q}_{t}$, which, for moderately anharmonic potentials, still follows closely the exact Matsubara trajectory on the FK surface - see Fig. 2. As a result of Eq. (33), the entire quantum Boltzmann distribution is conserved, provided the dynamics of $Q_{0, t}$ is generated by a quantum-Boltzmann-conserving method such as (T)RPMD or CMD (as in the planetary mode1 ${ }^{18}$ ). From this it follows that the planetary model gives the same result as CMD (within the FK approximation) for linear Kubo TCFs.

\section{PRACTICALITIES}

\section{A. Two practical modifications}

To test the planetary model on large systems we need to find a practical way to evaluate $V_{a^{2}}\left(Q_{0}\right)$ and $\Omega^{2}\left(Q_{0}\right)$ which bypasses the non-linear simultaneous Eqs. (24) and (25). ${ }^{35}$ Using these equations, we can write

$$
m \Omega^{2}\left(Q_{0}\right)=\frac{1}{\sqrt{2 \pi a^{2}}} \int_{-\infty}^{\infty} d q V^{\prime \prime}(q) e^{-\left(Q_{0}-q\right)^{2} / 2 a^{2}}
$$


which is an integral of the second derivative of $V(q)$ over a locally harmonic approximation to the Matsubara fluctuations around the centroid. This suggests that a similarly optimal local frequency, $\bar{\Omega}\left(Q_{0}\right)$, can be obtained by integrating over the exact Matsubara fluctuations to give

$$
m \bar{\Omega}^{2}\left(Q_{0}\right)=\lim _{N \rightarrow \infty} \frac{1}{(2 \pi \hbar)^{N} z_{N}\left(Q_{0}\right)} \int d \mathbf{p} \int d \mathbf{q} U_{N}^{\prime \prime}(\mathbf{q}) e^{-\beta R_{N}(\mathbf{p}, \mathbf{q})} \delta\left(\mathbf{q}, Q_{0}\right)
$$

with

$$
\begin{aligned}
U_{N}^{\prime \prime}(\mathbf{q}) & =\frac{1}{N} \sum_{i=1}^{N} V^{\prime \prime}\left(q_{i}\right) \\
\delta\left(\mathbf{q}, Q_{0}\right) & =\delta\left(\frac{1}{N} \sum_{i=1}^{N} q_{i}-Q_{0}\right) \\
z_{N}\left(Q_{0}\right) & =\frac{1}{(2 \pi \hbar)^{N}} \int d \mathbf{p} \int d \mathbf{q} e^{-\beta R_{N}(\mathbf{p}, \mathbf{q})} \delta\left(\mathbf{q}, Q_{0}\right) .
\end{aligned}
$$

Using integration by parts (as applied in ref. 36 to Eq. (34)) and converting to normal modes, we can eliminate the costly Hessian to give

$$
\begin{aligned}
m \bar{\Omega}^{2}\left(Q_{0}\right) \simeq \lim _{N \rightarrow \infty} & \frac{1}{(2 \pi \hbar)^{N} z_{N}\left(Q_{0}\right)} \frac{\beta_{N}}{N-1} \int d \mathbf{p} \int d \mathbf{q} e^{-\beta R_{N}(\mathbf{p}, \mathbf{q})} \delta\left(\mathbf{q}, Q_{0}\right) \\
& \times \sum_{n}^{\prime}\left[F_{n}^{2} / N-m \omega_{n}^{\prime 2} Q_{n} F_{n}\right]
\end{aligned}
$$

where $F_{n}=-\sum_{l=1}^{N} T_{l n} V^{\prime}\left(q_{l}\right)$ is the force along $Q_{n}$. Figure 3 shows that $\bar{\Omega}\left(Q_{0}\right)$ is an excellent approximation to $\Omega\left(Q_{0}\right)$ for the one-dimensional test system used to generate Fig. 2. Similar close agreement was also found for two-dimensional 'Champagne bottle' models, $\frac{31}{11}$ except in regions close to the repulsive wall, in which FK approximation itself is unlikely to be reliable. It thus seems reasonable to bypass the iterative equations by calculating $\bar{\Omega}\left(Q_{0}\right)$ in place of $\Omega\left(Q_{0}\right)$. The integral in Eq. (39) is not cheap but is manageable (taking about the same time as a CMD calculation, since it involves the sampling of the fluctuations about the centroid).

The other potential bottleneck is the evaluation of the centroid dynamics. In the original planetary model, this was done using CMD, with the force given by $-\partial V_{a^{2}}\left(Q_{0}\right) / \partial Q_{0}$. The analogous approach, with $\bar{\Omega}\left(Q_{0}\right)$ in place of $\Omega\left(Q_{0}\right)$ in Eqs. (24) and $25, \frac{37}{\text { would be }}$ difficult to implement, because the stochastic errors in $\bar{\Omega}\left(Q_{0}\right)$ could make the CMD trajectories unstable. However, since the centroid trajectory is independent of the planetary 
fluctuations, we are free to use any quantum-Boltzmann-conserving dynamics to generate $Q_{0, t}$. We propose the use of the TRPMD method with the exact (i.e. non-FK) potential $V$. The TRPMD method is cheap and efficient, and avoids the curvature errors which would affect CMD calculations on the same surface, $\frac{\sqrt[38]{389}}{i t s}$ drawback is that the Langevin thermostat broadens artificially the spectral lines. Figure 4 compares $\tilde{q}_{t}$ trajectories obtained using both the approximations of this Section (Eq. (39) and TRPMD) with the exact Matsubara trajectories on the FK surface (for the same one-dimensional model used to obtain Figs. 2 and 3); the two sets of trajectories remain in good agreement.

\section{B. Generalization to multi-dimensions}

The multi-dimensional analogue of Eq. (39) for a $D$-dimensional system is

$$
\begin{aligned}
\bar{\Omega}_{a b}^{2}\left(\mathbf{Q}_{0}\right) \simeq \lim _{N \rightarrow \infty} & \frac{1}{(2 \pi \hbar)^{D N}} \frac{\beta_{N}}{N-1} \int d \mathbf{p} \int d \mathbf{q} e^{-\beta R_{N}(\mathbf{p}, \mathbf{q})} \delta\left(\mathbf{q}, \mathbf{Q}_{0}\right) \\
& \times \sum_{n}^{1}\left[\frac{F_{n}^{a} F_{n}^{b}}{N \sqrt{m_{a} m_{b}}}-\omega_{n}^{\prime 2} \sqrt{\frac{m_{a}}{m_{b}}} Q_{n}^{a} F_{n}^{b}\right],
\end{aligned}
$$

where $F_{n}^{a}=-\sum_{l=1}^{N} T_{l n} \partial V(\mathbf{q}) / \partial q_{l}^{a}$ is the force on the $n^{\text {th }}$ normal mode coordinate that pertains to the $a^{\text {th }}$ dimension and

$$
\delta\left(\mathbf{q}, \mathbf{Q}_{0}\right)=\prod_{a=1}^{D} \delta\left(\frac{1}{N} \sum_{i=1}^{N} q_{i}^{a}-Q_{0}^{a}\right),
$$

where $\mathbf{q}_{i}$ is the position of the $i^{\text {th }}$ replica of the system. The corresponding radius of gyration matrix $A \equiv A\left(\mathbf{Q}_{0}\right)$ is given by

$$
A^{2}=\frac{1}{\beta} \bar{\Omega}^{-2}\left[\frac{\beta \hbar \bar{\Omega}}{2} \operatorname{coth}(\beta \hbar \bar{\Omega} / 2)-1\right],
$$

where $\bar{\Omega} \equiv \bar{\Omega}\left(\mathbf{Q}_{0}\right)$. (Note that we could alternatively have obtained $A$ as a direct integral of the fluctuations over $\tilde{q}^{2}$, by analogy with Eq. (35), but the $A$ of Eq. (42) has the advantage of being simultaneously diagonalizable with $\bar{\Omega}$ ). Defining the dimensionless coordinates $\overline{\mathbf{q}}=A^{-1} \tilde{\mathbf{q}}_{M}$ and $\overline{\mathbf{p}}=(A \bar{\Omega})^{-1} \tilde{\mathbf{p}}_{M}$ in terms of the mass-weighted fluctuation positions and momenta $\tilde{\mathbf{q}}_{M}$ and $\tilde{\mathbf{p}}_{M}$, we find the following multi-dimensional equations of motion, $\frac{19131}{1,1}$

$$
\begin{aligned}
& \dot{\overline{\mathbf{q}}}=\bar{\Omega} \overline{\mathbf{p}} \\
& \dot{\overline{\mathbf{p}}}=-\bar{\Omega} \overline{\mathbf{q}} .
\end{aligned}
$$


The real part of the planetary model dipole-moment autocorrelation function for a linear dipole moment is

$$
\begin{aligned}
c_{\mu \mu}^{[R]}(t)= & \widetilde{C}_{\mu \mu}(t)+\frac{1}{(2 \pi \hbar)^{D}} \int d \mathbf{P}_{0} \int d \mathbf{Q}_{0} \rho\left(\mathbf{P}_{0}, \mathbf{Q}_{0}\right) \frac{1}{(2 \pi)^{D} \operatorname{det}\left(\bar{\Omega} A^{2}\right)} \\
& \times \int d \tilde{\mathbf{p}}_{M} \int d \tilde{\mathbf{q}}_{M} e^{-\frac{1}{2} \tilde{\mathbf{q}}_{M}^{T} A^{-2} \tilde{\mathbf{q}}_{M}-\frac{1}{2} \tilde{\mathbf{p}}_{M}^{T}(\bar{\Omega} A)^{-2} \tilde{\mathbf{p}}_{M}} \boldsymbol{\mu}(\tilde{\mathbf{q}}) \cdot \boldsymbol{\mu}\left(\tilde{\mathbf{q}}_{t}\right),
\end{aligned}
$$

where $\widetilde{C}_{\mu \mu}(t)$ is the TRPMD dipole-moment autocorrelation function, $\rho\left(\mathbf{P}_{0}, \mathbf{Q}_{0}\right)$ is the exact centroid distribution, and $\widetilde{\mathbf{q}}_{t}$ results from evolution of $\widetilde{\mathbf{q}}$ through Eqs. (43) and (44) for time $t$.

\section{TESTS ON THE INFRARED SPECTRUM OF WATER}

We simulated gas-phase and condensed-phase q-TIP4P/F water at a variety of thermodynamic conditions to calculate infrared absorption spectra. The q-TIP4P/F water potential

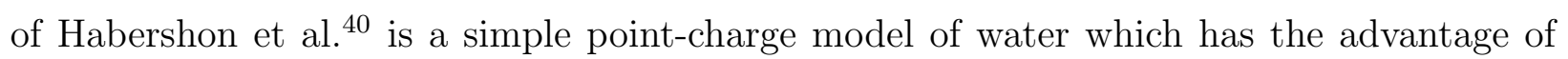
being cheap in comparison with other more sophisticated water potentials (e.g. TTM3-F, 41 PS, $\stackrel{42}{\text { MB-pol }[3,45}$ ), while still reproducing important quantum properties of water in the condensed phase. There are nine parameters in the model, four of which concern the intermolecular part of the potential and the other five concern the intramolecular part. The intermolecular part of the potential is identical to the TIP4P/2005 model of Abascal and Vega ${ }^{46}$ The intramolecular part of the potential comprises a harmonic $\mathrm{HOH}$ bend angle term and a fourth-order expansion of a Morse potential for the $\mathrm{OH}$ covalent bond stretch. The five parameters for the intramolecular part of the potential were optimised by Habershon et al. in a set of path integral calculations to give good agreement with experimental data for

liquid structure, self-diffusion constant and infrared absorption frequencies. All nine of the parameters are given in ref. 40 .

As is well known, the product of the Beer-Lambert absorption coefficient $\alpha(\omega)$ and the frequency-dependent refractive index $n(\omega)$ of a liquid with dipole-moment operator $\hat{\mu}$ is 47

$$
n(\omega) \alpha(\omega)=\frac{\pi \beta \omega^{2}}{3 c V \epsilon_{0}} \widetilde{G}_{\mu \mu}(\omega),
$$

where $\widetilde{G}_{\mu \mu}(\omega)$ is the Fourier transform of the quantum-Kubo dipole-moment autocorrelation function. Use of the relation

$$
\widetilde{G}_{A B}(\omega)=\frac{\beta \hbar \omega}{2} \operatorname{coth}\left(\frac{\beta \hbar \omega}{2}\right) G_{A B}^{[R]}(\omega)
$$


(obtained from Eq. (3)) allows us to rewrite this expression as,

$$
n(\omega) \alpha(\omega)=\frac{2 \pi \omega}{3 \hbar c V \epsilon_{0}} \tanh (\beta \hbar \omega / 2) G_{\mu \mu}^{[R]}(\omega),
$$

where $G_{\mu \mu}^{[R]}(\omega)$ is the Fourier transform of the real part of the standard quantum dipolemoment autocorrelation function. As mentioned in the Introduction, we use the planetary model to calculate the standard quantum TCF Eq. (48) and the TRPMD and CMD methods to calculate the Kubo TCF Eq. (46), in order to compare the fluctuation dynamics approximated by the planets with the centroid dynamics approximated by TRPMD or CMD. Note that when comparing with classical simulations, we followed the usual practice of comparing the Fourier transform of the classical dipole-moment autocorrelation function with Eq. (46), since this gives the exact result in the harmonic limit for a linear dipole-moment operator $\stackrel{47}{4}$

\section{A. Gas-phase water}

As a preliminary to the tests on liquid water and ice, we applied the planetary model to gas-phase water, taking a single $\mathrm{q}-\mathrm{TIP} 4 \mathrm{P} / \mathrm{F}$ molecule as the potential surface and using a linear approximation to the dipole-moment operator. The TCFs were artificially damped using functions of the form

$$
w(t ; \Delta t)=\left\{\begin{array}{ll}
\frac{1}{2}[\cos (\pi t / \Delta t)+1] & |t| \leq \Delta t \\
0 & \text { otherwise }
\end{array},\right.
$$

with $\Delta t$ chosen in accordance with the time taken for the TRPMD dipole-moment autocorrelation function to decorrelate in liquid water at $150 \mathrm{~K}(\Delta t=500 \mathrm{fs}), 300 \mathrm{~K}(400 \mathrm{fs})$ and $600 \mathrm{~K}$ (300 fs). The advantage of gas-phase water is that we can compare the planetary results directly with exact quantum results, which were calculated using the DVR3D package of Tennyson et al ${ }^{48}$ Further details of the gas-phase simulations are given in the supplementary material.

The infrared absorption spectra for gas-phase q-TIP4P/F water at $150 \mathrm{~K}, 300 \mathrm{~K}$ and $600 \mathrm{~K}$ are shown in Fig. 5. The insets show the stretch band between $3300 \mathrm{~cm}^{-1}$ and $4250 \mathrm{~cm}^{-1}$. The first point to note is that the classical stretch band is shifted to the blue at all temperatures (and by some $100 \mathrm{~cm}^{-1}$ relative to the quantum stretch band at $150 \mathrm{~K}$ ). This relative shift is accounted for by the significant zero-point energy of the anharmonic $\mathrm{OH}$ covalent bond and 
highlights the importance of a correct description of the quantum Boltzmann statistics in water. ${ }^{2}$ The planetary model and TRPMD stretch bands are much closer to the quantum result at the three plotted temperatures because they correctly account for the quantum Boltzmann statistics.

The second point to note is the emergence of a shoulder in the rotation band for the planetary model at $300 \mathrm{~K}$, and the emergence of a spurious peak between the rotation and bend bands at $150 \mathrm{~K}$. We found that setting the lowest six eigenvalues of the frequency matrix Eq. (40) to zero removed the shoulder and spurious peak. These are therefore caused by the harmonic description of the free rotation of the water molecule. The effect becomes more pronounced as the temperature decreases for two reasons. First, the spurious frequencies associated with rotation increase because ring polymers in the estimator of the frequency matrix become more delocalised along the rotation coordinates. Second, the weight of the planetary (fluctuation) contribution to the dipole-moment autocorrelation function of Eq. (45) increases relative to the centroid contribution. This is seen as a loss of absorbance of the rotational band in the correct region, which results from the adequate TRPMD description of rotation, and an increase in absorbance of the spurious peak. These results strongly suggest that the planetary model infrared absorption spectra should not be trusted for low frequencies.

However, the stretch and bend bands predicted by the planetary model are in excellent agreement with the quantum results, in position, absorbance and lineshape, even at 150K where the rotation band is most poorly described. One might expect to see erroneous features arising from spurious coupling to the incorrect planetary model description of the rotational dynamics, but these turned out to be extremely small (with the biggest effect being a spurious overtone band at about $3000 \mathrm{~cm}^{-1}$, which is too small to see in Fig. 5, but is just visible if the spectra are calculated using the original FK version of the planetary mode $\left.{ }^{(31}\right)$. This promising level of agreement suggests that the planetary model is able to give a good description of the stretches and bends at temperatures sufficiently low that TRPMD broadens the lineshapes (reducing the absorbance by almost a factor of two) ${ }^{49}$ and CMD and RPMD also give errors 50151 Note, however, that the spurious coupling just mentioned might be a problem in other systems, or in applications to non-linear TCFs. 


\section{B. Liquid water and ice}

We calculated classical, TRPMD and planetary-model infrared absorption spectra for qTIP4P/F hexagonal ice at 150K, liquid water at 300K and (compressed) liquid water at 600K at the experimental densities. To calculate these results we used the i-PI package of Ceriotti et al. with the included q-TIP4P/F driver. ${ }^{[52}$ We used periodic boundary conditions with a simulation box containing 96 water molecules for hexagonal ice and 128 water molecules for the liquid at $300 \mathrm{~K}$ and $600 \mathrm{~K}$. To accelerate convergence in the planetary calculations, we found it essential to take the Hadamard product of the approximate FK effective frequency matrix Eq. (40) with the following screening matrix,

$$
S_{a b}=\exp \left(-\gamma^{2} r_{a b}^{2}\right)
$$

where $\gamma$ is an adjustable parameter and $r_{a b}$ is the distance between the centroids that pertain to the $a^{\text {th }}$ and $b^{\text {th }}$ dimensions. For each condensed-phase system, we found that a value of $\gamma=0.10$ a.u. $^{-1}$ was sufficient to give the same infrared absorption spectrum as a smaller value. Reducing $\gamma$ has the advantage of requiring shorter centroid-constrained TRPMD trajectories to evaluate Eq. (35) (and is possible because the randomness in the Hessian matrix localises the normal modes to within a radius of $\gamma^{-1}$ ). Additional computational details of the condensed-phase q-TIP4P/F simulations, including the convergence of the

planetary spectra with respect to $\gamma$, are given in the supplementary material. We also compare with CMD infrared absorption spectra which are taken from ref. 53 .

The results of our simulations of condensed-phase q-TIP4P/F water are shown in Fig. 6. It is immediately noticeable that the planetary model does better for the libration band in the liquid water and ice spectra than it does for the rotational band in the gas-phase spectra (Fig. 5). At $150 \mathrm{~K}$ the planetary model libration band is shifted to the blue and shows a shoulder (the underlying TRPMD peak) but has not bifurcated as it has in the gas-phase case. Clearly, the harmonic approximation is less crude for hindered rotation in the condensed phase than for free rotation in the gas phase.

Except for the libration band, the relative positions and absorbances of the planetary model and TRPMD bands are very similar in the gas-phase and condensed-phase spectra. For example, at $150 \mathrm{~K}$ the absorbance of the planetary model stretch band is approximately twice that of TRPMD for both the gas-phase and condensed-phase infrared absorption spectra. Likewise, the positions of the planetary model and TRPMD stretch bands are in 
very good agreement at $150 \mathrm{~K}$ in both figures; the maximum of the planetary model stretch band lies approximately $10 \mathrm{~cm}^{-1}$ to the blue of the TRPMD maximum. ${ }^{54}$ By comparing with the exact quantum result for the single water molecule, we were able to determine that the planetary model provides a good representation of the stretch band for that system at $150 \mathrm{~K}$. We are unable to make such a comparison with an exact quantum q-TIP4P/F condensed-phase infrared absorption spectrum because the calculation of such a result is infeasible. However, it is reassuring to see that the planetary model and TRPMD stretch bands respond consistently to changes in the composition of the system. This observation leads us to speculate that the planetary model provides a good representation of the stretch band in both the condensed-phase and gas-phase systems, including at 150K.

\section{Motional narrowing in liquid water}

An interesting question is the extent to which the motion of the centroid affects the planetary model spectra; i.e. to what extent are the spectra in Fig. 6 simply a reflection of the static distribution of the local frequencies (i.e. the square roots of the eigenvalues of $\Omega^{2}\left(\mathbf{Q}_{0}\right)$ in Eq. 40$)$ ?

To answer this question, we repeated the planetary model simulations with the distribution of the centroids held static (following sampling by a ring-polymer master trajectory). This gives a static distribution of $\Omega^{2}\left(\mathbf{Q}_{0}\right)$ and the radius of gyration matrix $A^{2}\left(\mathbf{Q}_{0}\right)$. The infrared absorption spectrum that resulted from the planetary (fluctuation) part of Eq. (45) at $300 \mathrm{~K}$ is shown in Fig. 7. As is clear from the figure, this modification has a drastic effect which suggests that the planetary model spectra are not simply a reflection of the static distribution of frequencies. In particular, the bend and stretch bands are distorted through broadening and appear to no longer hold a Lorentzian lineshape. The stretch band is of a broad Gaussian shape that is some three to four times wider than the full-width-at-halfmaximum of the planetary stretch band in Fig. 6. We found this modification to have the same effect at $150 \mathrm{~K}$ and $600 \mathrm{~K}$ as well (results omitted).

Motional narrowing is the relevant theory for describing this phenomenon. In the absence of a time-dependent frequency matrix, the dynamics of the planets can be mapped onto an exact harmonic dynamics with each eigenfrequency constant in time. This results in the broad bands in Fig. 7 that directly reflect the static distribution of eigenfrequencies. 
However, with a time-dependent frequency matrix, the modulation of each eigenfrequency can sharpen these peaks.

The physics of motional narrowing in this context is described by Kubo's stochastic theory of lineshape ${ }^{[55}$ Kubo identifies two important quantities for the effect of frequency modulation on lineshapes by a stationary process. These are the time-independent standard deviation of the frequency and its correlation time. For fast modulation of the frequency, the spectrum of an oscillator that moves under the stochastic frequency shows motional narrowing: the lineshape becomes sharp with Lorentzian form. In the opposite limit, the lineshape is a direct reflection of the random distribution of frequencies. To reach this conclusion, Kubo assumes that the environment, as experienced by the system through the stochastic frequency, is insensitive to the motion of the system. This is precisely what happens in the planetary model, where the vibrational motion of the centroid can be identified with the environment and the planet with the system. We therefore conclude that the planetarymodel infrared absorption spectra show the phenomenon of motional narrowing as a result of the unidirectional interaction of the planet with the centroid through the time-dependent frequency matrix $\Omega\left(\mathbf{Q}_{0, t}\right)$.

Of course, the standard deviation of the frequency is a static property and the correlation time is a zero-frequency property of the system. It is well known that CMD, RPMD and TRPMD provide a consistent description of zero-frequency properties like the self-diffusion constant ${ }^{56}$ This suggests that for calculation of infrared absorption spectra, all of these methods would reproduce the effect of motional narrowing, and to the same extent, and the question of which in particular to use for the planetary model reduces to one of convenience. In other words, all three of the methods when used with the planetary model are expected to give very similar descriptions of the fluctuation dynamics.

To investigate further how well the planetary model describes the narrowing of the stretch band, we compare the stretch bands of the CMD, TRPMD and planetary-model condensedphase results at 300K and 600K in Fig. 8. To aid the comparison of lineshapes and intensities, we shifted the CMD stretch bands to align their median values with the planetary model results. As is clear from Fig. 8, the CMD and planetary-model lineshapes and intensities are in close agreement at both temperatures. On the other hand, the TRPMD lineshape is noticeably distorted at $300 \mathrm{~K}$ because of the thermostatting of the fluctuation modes. Although the absolute positions of the planetary model and CMD stretch bands differ, 
CMD and the planetary model appear to give an equivalent description of the structure of the stretch band for $\mathrm{q}-\mathrm{TIP} 4 \mathrm{P} / \mathrm{F}$ at temperatures exceeding $300 \mathrm{~K}$, despite being based on different approximations. This is a promising result for the planetary model, since previous CMD calculations using the TTM3-F water model gave lineshapes that were similar to the experimental lineshapes. ${ }^{17}$ The physical picture provided by the planetary model, in which the fluctuations are motionally narrowed by the vibrations of the centroid, is thus probably correct at 300 and $600 \mathrm{~K}$, and can probably also be trusted at $150 \mathrm{~K}$ (where the CMD stretch band is distorted and redshifted by the curvature problem $50|51| 531$.

\section{CONCLUSIONS}

The planetary model has been shown here to be an approximation to Matsubara dynamics, which works well when the dynamics is reasonably harmonic and bound, but not otherwise. When it does work, the model successfully captures the Matsubara fluctuations around the centroid by means of a single, phase-less, particle - the planet (see Fig. 1). We also find that the Feynman-Kleinert approximation on which the approach is based can be modified slightly to avoid iterative equations. In combination with TPRMD, this approach makes the computational effort of the planetary model comparable to that of (partially adiabatic) CMD, $\frac{16}{16}$ and thus applicable to the condensed phase.

Calculations of the IR spectrum in liquid water and ice (using the q-TIP4P/F potential and a linear dipole approximation) bear these findings out, and are probably a good indication of the applicability of the model more generally: it is never highly accurate - all the positions of the spectral lines are shifted somewhat; and, as expected, it fails for the libration band (which is strongly anharmonic). However, the bend and stretch band-positions are acceptable, and the shapes of the bands are very good, indicating that the planets give a realistic physical picture of the bend and stretch fluctuation dynamics in water. This suggests that the planetary model can usefully be applied to other systems where this part of the spectrum is important, e.g. low-dimensional materials 57 and molecular adsorbates,$\sqrt[58]{57}$ or con-

centrated acid and base solutions $\sqrt[59]{61}$ (for which the spectra show an interesting continuum between the bend and stretch).

Subject to the caveats just mentioned, the planetary model is currently (to our knowledge) the only practical method able to approximate realistically the dynamics of quantum 
Boltzmann fluctuations around the centroid. The role of such fluctuations is at present little understood, and the planetary model therefore promises to yield new insights. For example, the tests on water reported here found that the bend and stretch bands in the spectrum of the fluctuations were motionally narrowed by the vibrations of the centroid.

More importantly, the ability to treat fluctuation dynamics should allow the planetary model to be applied to TCFs involving non-linear operators. Smith et al. have already used the model to calculate dynamic structure factors for liquid hydrogen and reported good agreement with experiment. ${ }^{19}$ Further work will be needed to determine the extent to which these calculations can be generalized to systems such as water, as a number of things that make the calculations possible for linear TCFs (including the absence of explicit centroid-planet cross terms, and the smallness of stretch-libration overtone bands) may cause difficulties for non-linear TCFs. Nonetheless, the results reported here show that the

planetary model can often get the fluctuation dynamics reasonably right, and its future development for non-linear TCFs therefore looks promising.

\section{SUPPLEMENTARY MATERIAL}

See supplementary material for computational details of the q-TIP4P/F simulations on water (gas-phase, liquid, and ice).

\section{ACKNOWLEDGMENTS}

It is a pleasure to thank Jens Poulsen for invaluable help during the early stages of the project, and David Manolopoulos for suggestions that led to the methodology described in Sec. IVA. We also thank Mariana Rossi for supplying the CMD results in Fig. 8. This work was funded by the UK Engineering and Physical Sciences Research Council. 


\section{Appendix A: The Feynman-Kleinert approximation}

Here we summarise the derivation of the Feynman-Kleinert (FK) approximation given in ref. 34. The aim of the FK approximation is to determine the functions $L\left(Q_{0}\right)$ and $\Omega\left(Q_{0}\right)$ in Eq. (21) which variationally optimise the free energy $F_{\mathrm{FK}}\left(Q_{0}\right)$ obtained by integrating out the locally harmonic fluctuations $\mathbf{Q}^{\prime}$, i.e.

$$
e^{-\beta F_{\mathrm{FK}}\left(Q_{0}\right)}=z^{\prime}\left(Q_{0}\right) e^{-\beta L\left(Q_{0}\right)},
$$

where

$$
\begin{aligned}
z^{\prime}\left(Q_{0}\right) & =\frac{1}{(2 \pi \hbar)^{M-1}} \int^{\prime} d \mathbf{P} \int^{\prime} d \mathbf{Q} e^{-\beta H_{0}(\mathbf{P}, \mathbf{Q})} e^{-\beta m \sum_{n}^{\prime} \omega_{n}^{2} Q_{n}^{2} / 2} \\
& =\gamma_{M} \frac{\beta \hbar \Omega / 2}{\sinh (\beta \hbar \Omega / 2)}
\end{aligned}
$$

with $\gamma_{M}=(2 \pi)^{1-M}\{[(M-1) / 2] !\}^{-2}$ and

$$
H_{0}\left(\mathbf{P}^{\prime}, \mathbf{Q}^{\prime}\right)=\sum_{n}^{\prime} \frac{P_{n}^{2}}{2 m}+\frac{m}{2} \Omega^{2}\left(Q_{0}\right) Q_{n}^{2} .
$$

In other words, one seeks the $L\left(Q_{0}\right)$ and $\Omega\left(Q_{0}\right)$ that make $F_{\mathrm{FK}}\left(Q_{0}\right)$ as close as possible to the exact free energy

$$
e^{-\beta F\left(Q_{0}\right)}=z^{\prime}\left(Q_{0}\right)\left\langle e^{-\beta \Delta H}\right\rangle_{\mathrm{FK}}
$$

where

$$
\begin{gathered}
\langle\ldots\rangle_{\mathrm{FK}}=\frac{1}{(2 \pi \hbar)^{M-1} z^{\prime}\left(Q_{0}\right)} \int^{\prime} d \mathbf{P} \int^{\prime} d \mathbf{Q} e^{-\beta H_{0}(\mathbf{P}, \mathbf{Q})} e^{-\beta m \sum_{n}^{\prime} \omega_{n}^{2} Q_{n}^{2} / 2} \ldots \\
\Delta H(\mathbf{Q})=U_{M}(\mathbf{Q})-\sum_{n}^{\prime} \frac{m}{2} \Omega^{2}\left(Q_{0}\right) Q_{n}^{2} .
\end{gathered}
$$

The variational principle employed is the Gibbs-Bogoliubov variation principle $e^{62}$

$$
\left\langle e^{-\beta \Delta H}\right\rangle_{\mathrm{FK}} \leq e^{-\beta\langle\Delta H\rangle_{\mathrm{FK}}},
$$

This equation implies that if we choose

$$
L\left(Q_{0}\right)=\langle\Delta H\rangle_{\mathrm{FK}}
$$

then we can make $F_{\mathrm{FK}}\left(Q_{0}\right)$ as close as possible to $F\left(Q_{0}\right)$ by minimizing

$$
W\left(Q_{0}\right)=-\frac{1}{\beta} \ln z^{\prime}\left(Q_{0}\right)+\langle\Delta H\rangle_{\mathrm{FK}}
$$


with respect to $\Omega\left(Q_{0}\right)$ at each value of $Q_{0}$.

To evaluate $\langle\Delta H\rangle_{\mathrm{FK}}$ one writes

$$
\begin{aligned}
\left\langle U_{M}\right\rangle_{\mathrm{FK}} & =\frac{1}{\beta \hbar} \int_{0}^{\beta \hbar} d \tau\langle V[q(\tau)]\rangle_{\mathrm{FK}} \\
& =\langle V(\bar{q})\rangle_{\mathrm{FK}},
\end{aligned}
$$

where $\bar{q}=q(0)$; this last step follows from the symmetry of $U_{M}(\mathbf{Q})$ under imaginary-timetranslation. Writing $\bar{q}=Q_{0}+\tilde{q}$ with $\tilde{q}$ given by Eq. 20 gives

$$
\begin{aligned}
\left\langle U_{M}\right\rangle_{\mathrm{FK}}= & \frac{1}{(2 \pi \hbar)^{M-1} z^{\prime}\left(Q_{0}\right)} \int^{\prime} d \mathbf{P} \int^{\prime} d \mathbf{Q} e^{-\beta H_{0}(\mathbf{P}, \mathbf{Q})} e^{-\beta m \sum_{n}^{\prime} \omega_{n}^{2} Q_{n}^{2} / 2} \\
& \times \frac{1}{2 \pi \hbar} \int_{-\infty}^{\infty} d \tilde{q} \int_{-\infty}^{\infty} d \zeta e^{i \zeta\left(\tilde{q}-\sqrt{2} \sum_{n=1}^{(M-1) / 2} Q_{-n}\right) / \hbar} V\left(Q_{0}+\tilde{q}\right)
\end{aligned}
$$

where now $\tilde{q}$ is to be treated as an independent variable (not as a function of $Q_{n}$ as elsewhere). Evaluating the Gaussian integrals in $P_{n}, Q_{n}$ and $\zeta$ gives

$$
\left\langle U_{M}\right\rangle_{\mathrm{FK}}=\frac{1}{\sqrt{2 \pi a^{2}}} \int d \tilde{q} V\left(Q_{0}+\tilde{q}\right) e^{-\tilde{q}^{2} / 2 a^{2}},
$$

where

$$
a^{2}=\frac{2}{m \beta} \sum_{n}^{\prime} \frac{1}{\Omega_{n}^{2}}
$$

and $\Omega_{n}^{2}=\Omega^{2}+\omega_{n}^{2}$. Using ${ }^{63}$

$$
\frac{1}{2} \operatorname{coth} \beta \hbar \Omega / 2-\frac{1}{\beta \hbar \Omega}=\frac{2 \Omega}{\beta \hbar} \sum_{n=1}^{\infty} \frac{1}{\Omega_{n}^{2}}
$$

we find that $a^{2}$ is as given in Eq. (25) and hence $\left\langle U_{M}\right\rangle_{\mathrm{FK}}=V_{a^{2}}\left(Q_{0}\right)$ of Eq. (23). Repeating the integration with $-m \Omega^{2} \sum_{n}^{\prime} Q_{n}^{2} / 2$ in place of $V(q)$ yields $-m \Omega^{2}\left(Q_{0}\right) a^{2} / 2$, from which we obtain

$$
W\left(Q_{0}\right)=-\frac{1}{\beta} \ln z^{\prime}\left(Q_{0}\right)+V_{a^{2}}\left(Q_{0}\right)-\frac{m}{2} \Omega^{2}\left(Q_{0}\right) a^{2}
$$

which corresponds to $L\left(Q_{0}\right)$ of Eq. (22).

Finally, differentiating $W\left(Q_{0}\right)$ with respect to $\Omega$ gives

$$
\frac{d W\left(Q_{0}\right)}{d \Omega\left(Q_{0}\right)}=\left[\frac{\partial}{\partial a^{2}} V_{a^{2}}\left(Q_{0}\right)-\frac{m}{2} \Omega^{2}\right] \frac{d a^{2}}{d \Omega}=0
$$


of which the minimum is 65

$$
\begin{aligned}
\Omega^{2}\left(Q_{0}\right) & =\frac{2}{m} \frac{\partial}{\partial a^{2}} V_{a^{2}}\left(Q_{0}\right) \\
& =\frac{1}{m} \frac{\partial^{2}}{\partial Q_{0}^{2}} V_{a^{2}}\left(Q_{0}\right),
\end{aligned}
$$

thus proving Eq. (24).

The variation principle Eq. (A7) ensures that the FK approximation works also at barriers (in the sense of providing a reasonable approximation to $F_{M}\left(Q_{0}\right)$ ). This means that, when $Q_{0}$ is located at a barrier, the FK approximation must yield either a real value for $\Omega\left(Q_{0}\right)$, or an imaginary value which is above the instanton cross-over temperature $e^{66 \mid 67}$ i.e. $|\Omega|<2 \pi / \beta \hbar$. Note that this property is not guaranteed to hold for the non-variational $\bar{\Omega}\left(Q_{0}\right)$ of Eq. 35 .

\section{Appendix B: Details of the one-dimensional calculations}

To calculate the results in Figs. 2 and 4 we generated each set of initial centroid positions and momenta through $250 \mathrm{fs}$ of Andersen-thermostatted ${ }^{69}$ dynamics (with 32 Matsubara modes) according to the equations of motion in Eqs. (26) and (27). To generate each set of initial fluctuation positions and momenta, we resampled $Q_{n}$ and $P_{n}(n \neq 0)$ according to

$$
\begin{aligned}
Q_{n} & \sim \mathcal{N}\left(0,1 /\left\{\beta m\left[\omega_{n}^{2}+\Omega^{2}\left(Q_{0}\right)\right]\right\}\right) \\
P_{n} & \sim \mathcal{N}\left(0, m \Omega^{2}\left(Q_{0}\right) /\left\{\beta\left[\omega_{n}^{2}+\Omega^{2}\left(Q_{0}\right)\right]\right\}\right),
\end{aligned}
$$

where $\mathcal{N}\left(\mu, \sigma^{2}\right)$ denotes a normal distribution of mean $\mu$ and variance $\sigma^{2}$, and $\Omega^{2}\left(Q_{0}\right)$ is the squared FK effective frequency. The equations $(\mathrm{B} 1)$ and $(\mathrm{B} 2)$ ensure that

$$
\begin{aligned}
& \lim _{M \rightarrow \infty} \sum_{n}^{\prime}\left\langle Q_{n}^{2}\right\rangle=a^{2}\left(Q_{0}\right) \\
& \lim _{M \rightarrow \infty} \sum_{n}^{\prime}\left\langle P_{n}^{2}\right\rangle=m^{2} \Omega^{2}\left(Q_{0}\right) a^{2}\left(Q_{0}\right),
\end{aligned}
$$

where the thermal averages $\langle\cdot\rangle$ are taken over the fluctuation modes only, and $a^{2}\left(Q_{0}\right)$ is the squared FK radius of gyration. The relative initial planet positions and momenta were then constructed from

$$
\begin{aligned}
& \widetilde{q}=\sqrt{2} \sum_{n=1}^{(M-1) / 2} Q_{-n} \\
& \widetilde{p}=\sqrt{2} \sum_{n=1}^{(M-1) / 2} P_{-n} .
\end{aligned}
$$


These initial conditions $\left(P_{0}, Q_{0}, \widetilde{p}, \widetilde{q}\right)$ were evolved according to the relevant equations of motion (i.e. Eq. (26) or FK CMD / TRPMD for the centroid motion and Eqs. (27) or (32) for the planetary motion) with the velocity Verlet algorithm for alternating propagation of the centroid and planet with a time step of 0.25 fs. To calculate the TRPMD centroid trajectories, we used the thermostatted ring-polymer propagation scheme and the recommended friction matrix described in ref. 13 .

\section{Appendix C: Integrating out the Matsubara phase}

Substituting $U_{\mathrm{FK}}(\mathbf{Q})$ into Eq. (18), and using $\bar{q}_{1}=Q_{0}+\tilde{q}$ and $\bar{q}_{1, t}=Q_{0, t}+\tilde{q}_{t}$, we obtain

$$
c_{q q}^{[\mathrm{R}]}(t) \simeq \frac{1}{2 \pi \hbar Z_{M}} \int d P_{0} \int d Q_{0}\left[Q_{0} Q_{0, t}+I\left(Q_{0}, P_{0}, t\right)\right]
$$

where

$$
I\left(Q_{0}, P_{0}, t\right)=\frac{1}{(2 \pi \hbar)^{M-1}} \int^{\prime} d \mathbf{P} \int^{\prime} d \mathbf{Q} e^{-\beta H_{0}(\mathbf{P}, \mathbf{Q})} e^{i \beta \theta_{M}(\mathbf{P}, \mathbf{Q})} \tilde{q} \tilde{q}_{t}(\tilde{p}, \tilde{q})
$$

Note that there are no cross terms between $Q_{0}$ and $\tilde{q}$ because these would give odd integrals in $\tilde{q}$ and $\tilde{p}$. To evaluate Eq. (C2), we insert Dirac-delta functions such that

$$
\begin{gathered}
I\left(Q_{0}, P_{0}, t\right)=\frac{1}{(2 \pi \hbar)^{M-1}} \int_{-\infty}^{\infty} d \tilde{p} \int_{-\infty}^{\infty} d \tilde{q} \tilde{q} \tilde{q}_{t}(\tilde{p}, \tilde{q}) J\left(\tilde{p}, \tilde{q}, Q_{0}, P_{0}\right) \\
J\left(\tilde{p}, \tilde{q}, Q_{0}, P_{0}\right)=\frac{1}{(2 \pi \hbar)^{2}} \int_{-\infty}^{\infty} d \eta \int_{-\infty}^{\infty} d \zeta \int^{\prime} d \mathbf{P} \int^{\prime} d \mathbf{Q} e^{-\beta H_{0}(\mathbf{P}, \mathbf{Q})} e^{i \beta \theta_{M}(\mathbf{P}, \mathbf{Q})} \\
\times e^{i \eta\left(\sum_{n}^{\prime} T_{1 n} P_{n}-\tilde{p}\right) / \hbar+i \zeta\left(\sum_{n}^{\prime} T_{1 n} Q_{n}-\tilde{q}\right) / \hbar},
\end{gathered}
$$

where now $\tilde{p}$ and $\tilde{q}$ are to be treated as independent variables (not as functions of $P_{n}$ and $Q_{n}$ as they are elsewhere). Integrating over the Gaussian integrals in $P_{n}, Q_{n}, \zeta$ and $\eta$, and using Eq. (A14), we obtain

$$
J\left(\tilde{p}, \tilde{q}, Q_{0}, P_{0}\right)=\left(\frac{2 \pi}{\beta}\right)^{M-1}\left(\prod_{n}^{\prime} \frac{1}{\Omega_{n}}\right) \frac{1}{2 \pi a^{2} m \Omega} e^{-\tilde{q}^{2} / 2 a^{2}} e^{-\tilde{p}^{2} / 2 m^{2} \Omega^{2} a^{2}} \tilde{q} \tilde{q}_{t} .
$$

Evaluating $Z$ in the same way, and using

$$
\frac{\beta \hbar \Omega / 2}{\sinh \beta \hbar \Omega / 2}=\prod_{n=1}^{\infty}\left[1+\left(\frac{\Omega}{\omega_{n}}\right)^{2}\right]^{-1}
$$


gives Eq. (29).

Note that the Gaussian integral over $\zeta$ in Eq. (C4) is divergent if $\Omega\left(Q_{0}\right)$ is imaginary, and hence the 'planetary' expression as derived here is in general not valid at barriers (unless the FK approximation returns a real value of $\Omega\left(Q_{0}\right)$ at the barrier).

\section{REFERENCES}

${ }^{1}$ W.H. Miller, J. Chem. Phys. 125, 132305 (2006).

${ }^{2}$ M. Ceriotti, W. Fang, P.G. Kusalik, R.H. McKenzie, A. Michaelides, M.A. Morales, and T.E. Markland, Chem. Rev. 116, 7529 (2016).

${ }^{3}$ T.J.H. Hele, M.J. Willatt, A. Muolo, and S.C. Althorpe, J. Chem. Phys. 142, 134103 (2015).

${ }^{4}$ T.J.H. Hele, M.J. Willatt, A. Muolo, and S.C. Althorpe, J. Chem. Phys. 142, 191101 (2015).

${ }^{5}$ W.H. Miller, J. Phys. Chem. A 105, 2942 (2001).

${ }^{6}$ J. Liu, Int. J. Quantum Chem. 115, 657 (2015).

${ }^{7}$ M.S. Church, S.V. Antipov, and N. Ananth, J. Chem. Phys. 146, 234104 (2017).

${ }^{8}$ J. Liu, J. Chem. Phys. 140, 224107 (2014).

${ }^{9}$ J. Liu and Z. Zhang., J. Chem. Phys. 144, 034307 (2016).

${ }^{10}$ I.R. Craig and D.E. Manolopoulos, J. Chem. Phys. 121, 3368 (2004).

${ }^{11}$ I.R. Craig and D.E. Manolopoulos, J. Chem. Phys. 123, 034102 (2005).

${ }^{12}$ S. Habershon, D.E. Manolopoulos, T.E. Markland and T.F. Miller III, Annu. Rev. Phys. Chem. 64, 387 (2013).

${ }^{13}$ M. Rossi, M. Ceriotti, and D.E. Manolopoulos, J. Chem. Phys. 140, 234116 (2014).

${ }^{14}$ N. Boekelheide, R. Salomón-Ferrer, and T.F. Miller III, Proc. Natl. Acad. Sci. 108, 16159 (2011).

${ }^{15}$ J. Cao and G.A. Voth, J. Chem. Phys. 101, 6168 (1994).

${ }^{16}$ T.D. Hone, P.J. Rossky and G.A. Voth, J. Chem. Phys. 124, 154103 (2006).

${ }^{17}$ F. Paesani, S.S. Xantheas, and G.A. Voth, J. Phys. Chem. B 113, 13118 (2009).

${ }^{18}$ K.K.G. Smith, J.A. Poulsen, G. Nyman, and P.J. Rossky, J. Chem. Phys. 142, 244112 (2015). 
${ }^{19}$ K.K.G. Smith, J.A. Poulsen, G. Nyman, A. Cunsolo, and P.J. Rossky, J. Chem. Phys. 142, $244113(2015)$.

${ }^{20}$ A. Horikoshi and K. Kinugawa, J. Chem. Phys. 122, 174104 (2005).

${ }^{21}$ A. Sinitskiy and G.A. Voth, J. Chem. Phys. 143, 094104 (2015).

${ }^{22}$ In the notation of Sec. III, the locally harmonic force on the 'pseudoparticle' is a function of $\mathbf{q}(\tau=0)$, whereas the force on the 'planet' is a function of $\mathbf{Q}_{0}$.

${ }^{23}$ R. Kubo, J. Phys. Soc. Japan 12, 570 (1957).

${ }^{24}$ D. Chandler and P.G. Wolynes, J. Chem. Phys. 74, 4078 (1981).

${ }^{25}$ M. Parrinello and A. Rahman, J. Chem. Phys. 80, 860 (1984).

${ }^{26}$ D.M. Ceperley, Rev. Mod. Phys. 67, 279 (1995).

${ }^{27}$ M.E. Tuckerman, Statistical Mechanics: Theory and Molecular Simulation (Oxford University Press, Oxford, 2010).

${ }^{28}$ T. Matsubara, Prog. Theor. Phys. 14, 351 (1955).

${ }^{29}$ Strictly speaking, the distinction is that the Matsubara modes give continuous distributions of points $q(\tau)$ whereas the jagged modes give discontinuous distributions. This is because there are $N(\rightarrow \infty)$ equally spaced intervals in $\tau$, and the length of the path $q(\tau)$ scales as $N^{0}$ for the Matsubara modes and $N$ for the jagged modes.

${ }^{30}$ The effective Planck's constant for the Matsubara modes is $\lim _{N \rightarrow \infty} \hbar / N=0$; see ref. 3 .

${ }^{31}$ M.J. Willatt, Ph.D. thesis, University of Cambridge, 2017.

${ }^{32}$ Identical results would be obtained using $q(\tau)$ at any other $0 \leq \tau<\beta \hbar$.

${ }^{33}$ Note that there can be no second-order coupling terms $Q_{n} Q_{n^{\prime} \neq n}$ because of the requirement that $U_{F K}(\mathbf{Q})$ be symmetric with respect to imaginary-time-translation.

${ }^{34}$ R.P. Feynman and H. Kleinert, Phys. Rev. A 34, 5080 (1986).

${ }^{35}$ Alternatively, one can seek efficient ways of dealing with the iterative solution of Eqs. (24) and (25); see ref. 36 .

${ }^{36}$ J.A. Poulsen, G. Nyman, and P.J. Rossky, J. Chem. Theory Comput. 2, 1482 (2006).

${ }^{37}$ This use of the exact centroid distribution with the FK description of the fluctuations has also been proposed in a different context; see Appendix A in: J. Liu and W.H. Miller, J. Chem. Phys. 131, 074113 (2009).

${ }^{38} \mathrm{An}$ alternative approach could be to expand the potential as a linear combination of Gaussian functions, $\frac{19 \mid 36}{10}$ allowing $\bar{\Omega}^{2}\left(Q_{0}\right)$ to be evaluated analytically. 
${ }^{39}$ The FK CMD curvature error is greatly reduced since the FK approximation is locally harmonic; some curvature effects remain in the effective frequencies $\Omega$.

${ }^{40}$ S. Habershon, T.E. Markland, and D.E. Manolopoulos, J. Chem. Phys. 131, 024501 (2009).

${ }^{41}$ G.S. Fanourgakis and S.S. Xantheas, J. Chem. Phys. 128, 074506 (2008).

${ }^{42}$ H. Partridge and D. Schwenke, J. Chem. Phys. 106, 4618 (1997).

${ }^{43}$ V. Babin, C. Leforestier, and F. Paesani, J. Chem. Theory Comput. 9, 5395 (2013).

${ }^{44}$ V. Babin, G.R. Medders, and F. Paesani, J. Chem. Theory Comput. 10, 1599 (2014).

${ }^{45}$ G. R. Medders, V. Babin, and F. Paesani, J. Chem. Theory Comput. 10, 2906 (2014).

${ }^{46}$ J.L. Abascal and C. Vega, J. Chem. Phys. 123, 234505 (2005).

${ }^{47}$ S. Habershon, G.S. Fanourgakis, and D.E. Manolopoulos, J. Chem. Phys. 129, 074501 (2008).

${ }^{48}$ J. Tennyson et al., Comput. Phys. Commun. 163, 85 (2004).

${ }^{49}$ Note that there is a new, generalized, form of TRPMD, presented in another article in this special issue, which uses coloured noise to reduce considerably the artifactual broadening; see: M. Rossi, V. Kapil, and M. Ceriotti, J. Chem. Phys. 148, 102301 (2018).

${ }^{50}$ A. Witt, S.D. Ivanov, M. Shiga, H. Forbert, and D. Marx, J. Chem. Phys. 130, 194510 (2009).

${ }^{51}$ S.D. Ivanov, A. Witt, M. Shiga, and D. Marx, J. Chem. Phys. 132, 031101 (2010).

${ }^{52}$ M. Ceriotti, J. More, and D.E. Manolopoulos, Comput. Phys. Commun. 185, 1019 (2014).

${ }^{53}$ M. Rossi, H. Liu, F. Paesani, J. Bowman, and M. Ceriotti, J. Chem. Phys. 141, 181101 (2014).

${ }^{54}$ These positions are close to those predicted by the local monomer (LMon) method; see: Y. Wang and J.M. Bowman, J. Chem. Phys. 136, 144113 (2012).

${ }^{55}$ R. Kubo, Adv. Chem. Phys.: Stochastic Processes in Chemical Physics 15, 101 (1969).

${ }^{56}$ T.F. Miller III and D.E. Manolopoulos, J. Chem. Phys. 122, 184503 (2005).

${ }^{57}$ K.N. Kudin, B. Ozbas, H.C. Schniepp, R.K. Prud'homme, I.A. Aksay, and R. Car, Nano Lett. 8, 36 (2008).

${ }^{58}$ S.A. FitzGerald, K. Allen, P. Landerman, J. Hopkins, J. Matters, R. Myers, and J.L.C. Rowsell, Phys. Rev. B 77, 224301 (2008).

${ }^{59}$ S.T. Roberts, K. Ramasesha, P.B. Petersen, A. Mandal, and A. Tokmakoff, J. Phys. Chem. A 115, 3957 (2011). 
${ }^{60}$ S.T. Roberts, P.B. Petersen, K. Ramasesha, A. Tokmakoff, I.S. Ufimtsev, and T.J. Martinez, Proc. Natl. Acad. Sci. 106, 15154 (2009).

${ }^{61}$ M. Thämer, L. De Marco, K. Ramasesha, A. Mandal, and A. Tokmakoff, Science 350, 78 (2015).

${ }^{62}$ D. Chandler, Introduction to Modern Statistical Mechanics (Oxford University Press, New York, 1987).

${ }^{63}$ Equation 1.421(4) in ref. 64.

${ }^{64}$ I.S. Gradshteyn and I.M. Ryzhik, Table of Integrals, Series and Products, 6th ed. (Academic Press, San Diego, California, 2000).

${ }^{65} \mathrm{H}$. Kleinert, Path integrals in quantum mechanics, statistics, polymer physics, and financial markets, 4th ed. (World Scientific, 2006).

${ }^{66}$ J.O. Richardson and S.C. Althorpe, J. Chem. Phys. 131, 214106 (2009).

${ }^{67}$ J.O. Richardson, J. Chem. Phys. 144, 114106 (2016).

${ }^{68}$ Equation 1.431(2) in ref. 64.

${ }^{69}$ H.C. Andersen, J. Chem. Phys. 72, 2384 (1980). 


\section{FIGURES CAPTIONS}

FIG. 1. Schematic illustrating how the planetary model of ref. 18 approximates the dynamics of Matsubara fluctuations around the centroid. The 'planet' $\mathbf{q}(\tau=0)$ is a single point along the smooth imaginary-time distribution $\mathbf{q}(\tau), 0 \leq \tau<\beta \hbar$ (grey dashes). The Matsubara dynamics of $\mathbf{q}(\tau)$ is approximated by locally harmonic fluctuations around the centroid position $\mathbf{Q}_{0}$, which decouples the dynamics of the planetary fluctuation coordinate $\tilde{\mathbf{q}}$, allowing the Matsubara phase to be integrated out.

FIG. 2. Matsubara fluctuation trajectories $\tilde{q}_{t}$ calculated at different levels of approximation for one-dimensional Feynman-Kleinert (FK) models of (a) an $\mathrm{OH}$ stretch and (b) an $\mathrm{OH}$ rotation, both at $300 \mathrm{~K}$ (see text for details). The approximations compared are: exact Matsubara dynamics on the FK surface (black), the locally harmonic approximation of Sec. IIIB (red), and the planetary model of ref. 18 (blue).

FIG. 3. Comparison of the directly-sampled effective frequency $\bar{\Omega}\left(Q_{0}\right)$ (blue) of Eq. 35 with the FK effective frequency $\Omega\left(Q_{0}\right)$ (dashed red), for the OH-stretch model of Fig. 2. Also shown is the classical frequency $\sqrt{V^{\prime \prime}\left(Q_{0}\right) / m}$ evaluated at the centroid (black).

FIG. 4. Matsubara trajectories calculated at different levels of approximation for the $\mathrm{OH}$-stretch model used in Fig. 2a. Panel (a) shows centroid trajectories $Q_{0}(t)$ calculated using exact Matsubara dynamics on the FK surface (black), the planetary model of ref. 18 (blue), and the modified planetary model of Sec. IVA (green). Panel (b) shows the corresponding Matsubara fluctuation trajectories $\tilde{q}_{t}$.

FIG. 5. Infrared absorption spectra for gas-phase q-TIP4P/F water, at $150 \mathrm{~K}, 300 \mathrm{~K}$ and $600 \mathrm{~K}$, calculated using the planetary model (black), and compared with the results of TRPMD (blue), exact quantum (dashed black) and classical (red) calculations. The absorbance is given in arbitrary units. The insets show the stretch band from $3300 \mathrm{~cm}^{-1}$ to $4250 \mathrm{~cm}^{-1}$. 
FIG. 6. Infrared absorption spectra for q-TIP4P/F hexagonal ice (150K), liquid water (300K) and compressed liquid water $(600 \mathrm{~K})$, calculated using the planetary model (black), and compared with the results of TRPMD (blue), CMD (green, taken from ref. 53) and classical (red) calculations.

FIG. 7. Infrared absorption spectra for q-TIP4P/F liquid water (300K), showing the effects of motional narrowing on the lineshapes. The planetary model (black), TRPMD (blue) and CMD (green) spectra are the same as in Fig. 6. The broad red spectrum was calculated using the planetary model, with the centroid positions held fixed (see Sec. VC).

FIG. 8. Stretch-band lineshapes for q-TIP4P/F liquid water (300K, solid lines) and compressed liquid water $(600 \mathrm{~K}$, dashed lines). The planetary model (black) and TRPMD (blue) spectra are the same as in Fig. 6. The CMD spectra of ref. 53 (green) have been shifted (but not scaled). 